\title{
EFFICIENT STOCHASTIC GRADIENT SEARCH FOR AUTOMATIC IMAGE REGISTRATION
}

\author{
Li, Q.; Sato, I. \& Murakami, Y. \\ GSJ/AIST - National Institute of Advanced Industrial Science and Technology, \\ Central 7, 1-1-1 Higashi, Tsukuba 305-8567, Japan \\ E-Mail: qi.li@aist.go.jp; isao.sato@aist.go.jp; y.murakami@aist.go.jp
}

\begin{abstract}
This paper focuses mainly on an efficient stochastic gradient search algorithm for the study of optimal registration transformation. A simultaneous perturbation stochastic approximation technique is successfully implemented on image registration by optimizing mutual information based similarity measures. The hill climbing search and simplex direct search are also conducted in the experiments for the comparative purpose. The registration experiments are associated with the pairs of optical sensor images, synthetic aperture radar images and medical multimodality images, which are misaligned by the rigid or affine transformations. The experimental results show that in general the stochastic gradient search yields significant improvements on the optimal solution over the conventional hill climbing and simplex direct search in terms of accuracy and robustness. The main contribution of this paper is the first accomplishment of an efficient stochastic gradient search strategy on the mutual information based automatic image registration.

(Extended paper from the International Symposium CompIMAGE - Coimbra, Portugal, 20-21 October 2006.)
\end{abstract}

Key Words: Image Registration, Optimization Technique, Stochastic Gradient, Mutual Information, Affine Transformation

\section{INTRODUCTION}

Image registration is the first key step for many image applications in the field of the computer vision, image aided medical operation and earth observation mission. Mathematically, image registration is defined as a simulation modelling which determines a mapping that is the best match of two or more images of the same scene acquired by different sensors, or taken by the same sensor at different times [1].

The core process of image registration is to optimize an object function defined by a similarity measure. The mutual information based similarity measure, which is proposed by Voila \& Wells [2] and Collignon et al. [3] independently, is popularly used in the current study. The choice to use this similarity measure is strongly based on the outstanding merits of the mutual information that it does not depend on any assumption on the image set and does not assume specific relationship between image intensities in different modalities. To apply the mutual information criterion for a realistic image registration, an important problem should be faced. That is how to efficiently and robustly find the global optima of the object function which is defined by mutual information. Our proposed automatic image registration diagram is shown in Fig. 1. It can be noted that the optimization module is the driver for the whole registration process. In practice, a local search algorithm is almost the first choice because most of imaging applications assume that the initial misregistration is relatively small enough. In the past research, many local search algorithms have been proposed [4]. In this paper, three local search algorithms, viz. hill climbing, Nelder-Mead simplex and stochastic gradient, are considered to solve the optimization problem of the image registration. The stochastic gradient search which is constructed by a simultaneous perturbation stochastic approximation 
(SPSA) technique firstly proposed by Spall [5] is proposed for optimizing the mutual information based similarity measure. The main difference between our work and the other researchers' [6-8] is that we firstly conclude three aspects of efficiency of the stochastic gradient search algorithm over the other two. Especially, we firstly and successfully applied it to optimize a mutual information based pseudo-metric for simultaneously registering the sixparameter affine transformation for the multimodality images.

The paper is organized as follows: Section 1 introduces the research background. Section 2 presents the two parts, mutual information based similarity measure and parametric transformation, associated with the automatic image registration. Section 3 describes the implementation of three local search algorithms. Section 4 presents some comparative experimental results and discussion. Section 5 gives some concluding remarks.

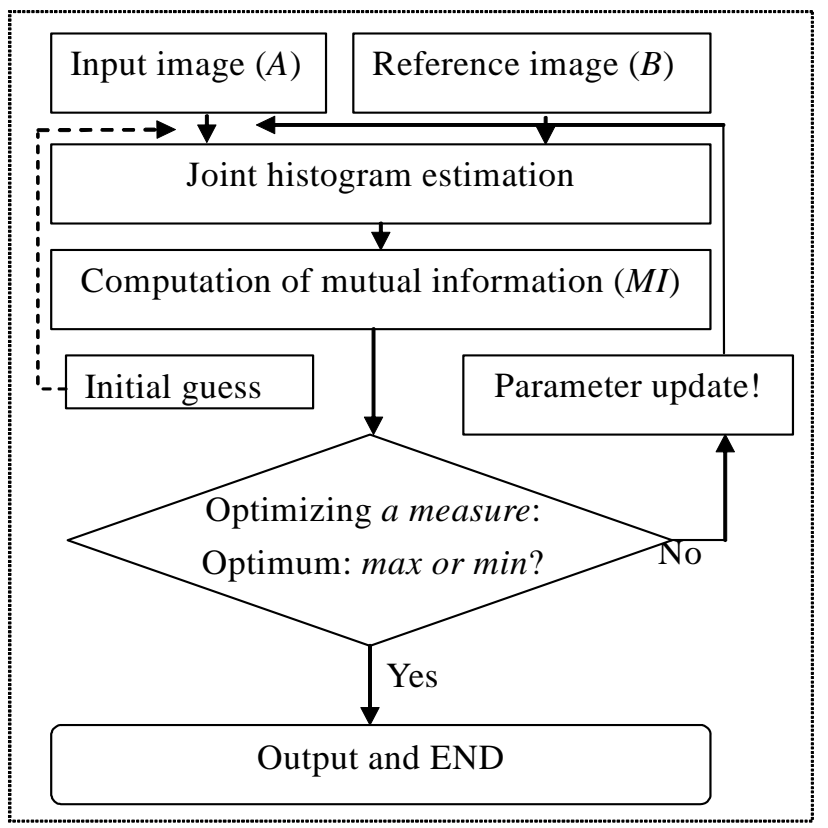

Figure 1: Image registration diagram.

\section{AUTOMATIC IMAGE REGISTRATION}

\subsection{Parametric transformation}

In our automatic registration study, the rigid (translation and/or rotation only) and affine transformations are considered to register the pairs of gray-scale images. The rigid transformation matrix can be written as:

$$
T_{p}^{\text {rigid }}=\left[\begin{array}{ccc}
\cos (\theta) & -\sin (\theta) & 0 \\
\sin (\theta) & \cos (\theta) & 0 \\
t x & t y & 1
\end{array}\right]
$$

where $(t x, t y)$ and $\theta$ denote the translation and rotation respectively.

The general affine transformation matrix can be written as:

$$
T_{p}^{a f f i n e}=\left[\begin{array}{lll}
a & b & 0 \\
c & d & 0 \\
e & f & 1
\end{array}\right]
$$


In the affine registration experiment, we decompose $T_{p}^{a f f i n e}$ into a product of scale, shear and rotations plus translations. The decomposition is listed as follows:

$$
\begin{aligned}
T_{p}^{\text {affine }}=\left[\begin{array}{lll}
a & b & 0 \\
c & d & 0 \\
0 & 0 & 0
\end{array}\right]+\left[\begin{array}{lll}
0 & 0 & 0 \\
0 & 0 & 0 \\
e & f & 1
\end{array}\right]=T_{p}^{\text {affine }, I}+T_{p}^{a f f i n e, I I} \\
T_{p}^{a f f i n e, I}=\left[\begin{array}{lll}
2^{s} & 0 & 0 \\
0 & 2^{s} & 0 \\
0 & 0 & 0
\end{array}\right] R(\theta)\left[\begin{array}{ccc}
2^{t} & 0 & 0 \\
0 & 2^{-t} & 0 \\
0 & 0 & 0
\end{array}\right] R(\phi) \\
R(\theta)=\left[\begin{array}{ccc}
\cos (\theta) & -\sin (\theta) & 0 \\
\sin (\theta) & \cos (\theta) & 0 \\
0 & 0 & 0
\end{array}\right] \\
R(\phi)=\left[\begin{array}{ccc}
\cos (\phi) & -\sin (\phi) & 0 \\
\sin (\phi) & \cos (\phi) & 0 \\
0 & 0 & 0
\end{array}\right]
\end{aligned}
$$

where $s$ and $t$ are scale and shear parameters, $\theta$ and $\phi$ are two rotation parameters, and $e$ and $f$ are two translation parameters.

The goal of image registration is to find the optimal parameters discussed above which define the relative position and orientation of the two sensed images in an efficient and robust way.

\subsection{Mutual information}

Based on the information theory [9], the definition of standard mutual information, $M I(A, B)$, of two random signals $A$ and $B$ can be calculated as follows:

$$
M I(A, B)=H(A)+H(B)-H(A, B)
$$

where $H(A)$ and $H(B)$ are the marginal entropies of $A$ and $B$, and $H(A, B)$ is their joint entropy. Considering $A$ and $B$ as two images (i.e., two $2 D$ signals), the mutual information is maximal when the two images are totally geometrically aligned by a certain transformation matrix. In our latter rigid registration experiments, the standard mutual information criterion is adopted. In practical applications, many pseudo metrics are educed from the standard mutual information for the different purposes. In our latter affine registration experiment, a normalized pseudo metric [10] is used, and it is defined as follows:

$$
p M I(A, B)=\frac{2 H(A, B)}{H(A)+H(B)}-1
$$

In the computation of mutual information, the marginal entropies and joint entropy can be calculated as follows:

$$
\begin{gathered}
H(A)=\sum_{a}-p_{A}(a) \log p_{A}(a) \\
H(B)=\sum_{b}-p_{B}(b) \log p_{B}(b) \\
H(A, B)=\sum_{a} \sum_{b}-p_{A, B}(a, b) \log p_{A, B}(a, b)
\end{gathered}
$$


where $P_{A}(a)$ and $P_{B}(b)$ are the marginal probability mass functions, and $P_{A, B}(a, b)$ is the joint probability mass function. These probability mass functions can be computed from the histogram of images as follows:

$$
\begin{gathered}
p_{A, B}(a, b)=\frac{h(a, b)}{\sum_{a} \sum_{b} h(a, b)} \\
p_{A}(a)=\sum_{b} p_{A, B}(a, b) \\
p_{B}(b)=\sum_{a} p_{A, B}(a, b)
\end{gathered}
$$

where $h$ is the joint histogram of the image pair. The value of $h(a, b)$ is the statistic numbers of corresponding pairs having intensity value $a$ in the input image $A$ and intensity value $b$ in the reference image $B$ [11]. In the whole experiments, the partial volume interpolation was used for the estimation of joint histogram.

\section{LOCAL SEARCH ALGORITHM}

Although the image registration can be mapped as a typical optimization problem, the interpolation artifact complicates the object function which is defined by the similarity measure, and also with the increase of the transformation parameters, the image registration becomes into a complicated non-convex optimization problem (see Fig. 2), so the efficient optimizer is very important during the iterated search of a better-quality solution. In this section, three local search algorithms are introduced for the registration study.

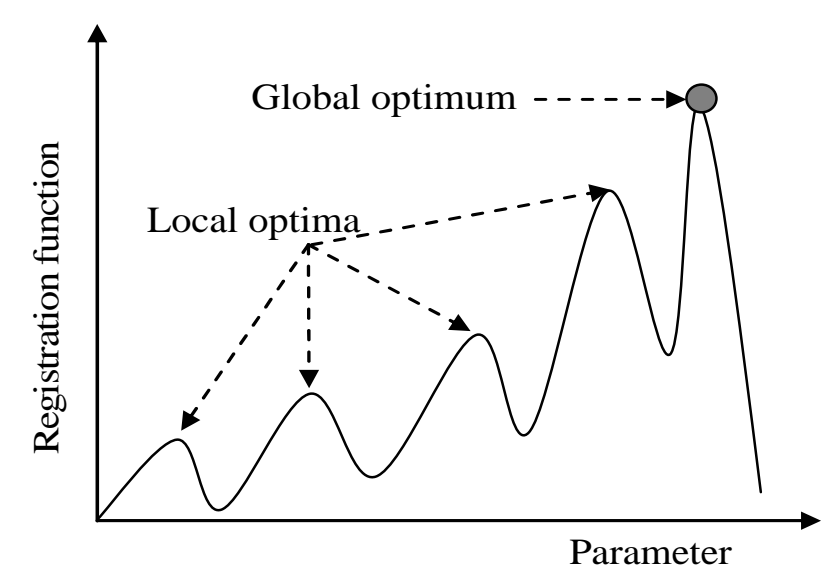

Figure 2: Non-convex registration function and the concept of local and global optima.

\subsection{Hill climbing search}

The hill climbing is probably the most basic local search algorithm where the current path is extended with a successor node which is closer to the solution than the end of the current path. It is easy to understand and implement. In simple hill climbing, the first closer node is chosen whereas in steepest ascent hill climbing all successors are compared and the closest to the solution is chosen. The algorithm terminates when there is no closer node. This may happen if it is gotten stuck in local maxima in the search space which are not solutions [12, 13].

\subsection{Simplex direct search}

The Nelder-Mead simplex direct search is a commonly used nonlinear optimization algorithm for minimizing or maximizing an objective function in a high-dimensional space [14]. The 
method uses the concept of a simplex, which is a polytope of $N+1$ vertices in $N$ dimensions: a line segment on a line, a triangle on a plane, a tetrahedron in three-dimensional space and so forth. It approximately finds a locally optimal solution to a problem with $N$ variables when the objective function varies smoothly. The search procedure terminates when the standard deviation of each transformation parameter of the final simplex is less than the residual settings.

\subsection{Stochastic gradient search}

The local stochastic gradient search accomplished in our study is based on the SPSA technique, which is firstly put forward by Spall. It becomes seriously popular for solving some challenging optimization problems. The prominent merit of this algorithm is that it does not require an explicit knowledge of the gradient of the object function, or measurements of this gradient. At each iteration, it only needs an approximation to the gradient via simultaneous perturbations. Especially, the gradient approximation is based on only two function measurements regardless of the dimensions of the search parameter space. The SPSA based stochastic gradient search is a very strong local optimizer, which can get through some local optima of the objective function to successfully find the global optima because of the stochastic nature of the gradient approximation [5]. The idiographic implementation of the standard SPSA technique on the image registration can be found in $[6,15]$.

\section{EXPERIMENTAL RESULTS AND DISCUSSION}

\subsection{Rotation transformation}

The first experiment is designed to register one parameter (rotation) transformation using a standard mutual information criterion. This is the simplest rigid transformation. Fig. 3 (i) and (ii) show a pair of the PALSAR (Phased Array type L-band Synthetic Aperture Radar) images to be registered. Fig. 3 (ii) is artificially generated from (i) by rotating 5 degrees clockwise with the cubic interpolation. Fig. 4 shows the mutual information as a registration function of the rotation angle in degrees with plotting the search trajectory to the global maximum. The initial guess starts from -5 degrees for each search algorithm. The search trajectory is shown as a dashed line and the current solution is shown as a circle in Fig. 4.

By observing Fig. 4, it can be found that the stochastic gradient search shows the best performance/cost balance to the rotation registration. The hill climbing search suffers from getting stuck at the local maxima. The simplex direct search can jump over the local maxima, but the computation of simplex costs too much time.

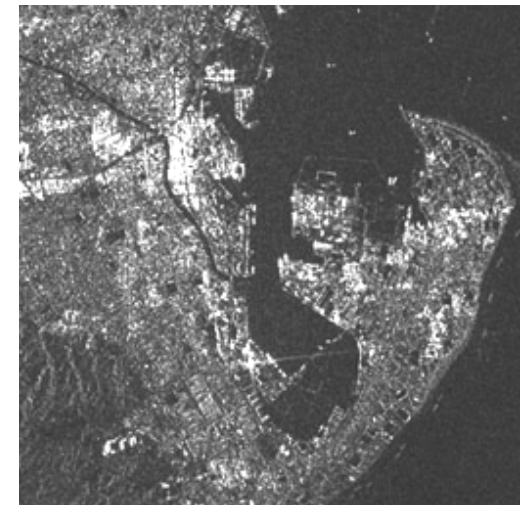

(i) PALSAR image

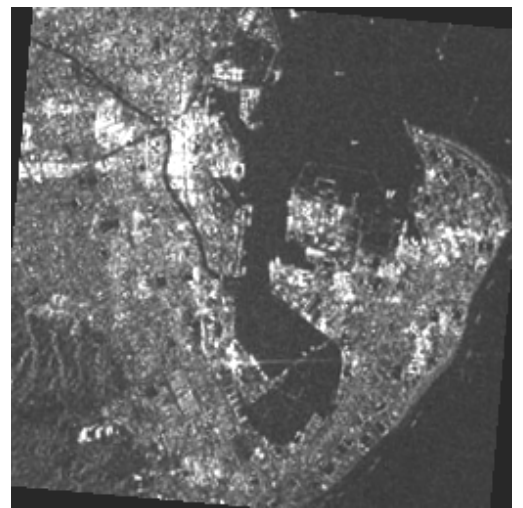

(ii) Transformed PALSAR image

Figure 3: PALSAR image pair (Location: Shizuoka city, Japan; Size: 256x256 pixel²; Date: 15-02-2006). 


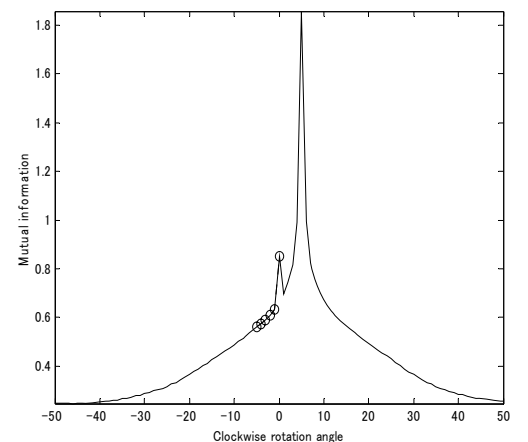

(i) Hill climbing search

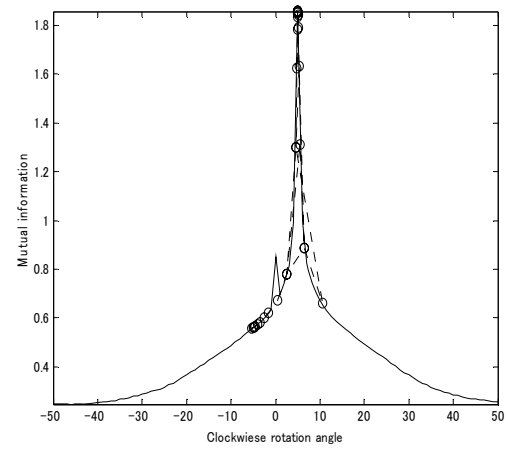

(ii) Simplex direct search

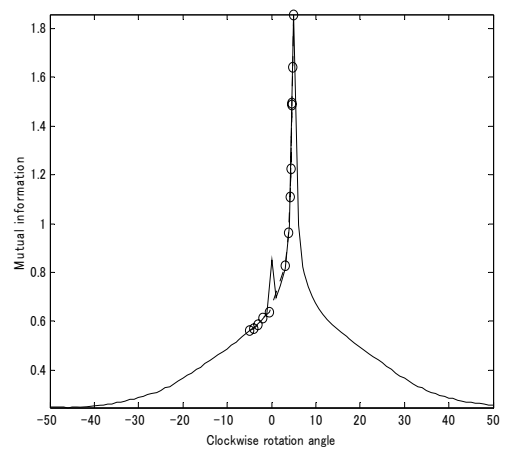

(iii) Stochastic gradient search

Figure 4: The registration function and the search trajectory.

\subsection{Translation transformation}

The second experiment is to register two ASTER (advanced spaceborne thermal emission and reflection radiometer) VNIR (visual near-infrared) images along two-direction translations using a standard mutual information criterion. The registered images are shown in Fig. 5 . The true solution of the image pair is $(0,7)$ if the image without cloud contamination is regarded as the template and the bottom left corner is regarded as the coordinate origin.

In order to test the performance of three local search algorithms, ten initial guesses near the true solution are randomly generated in the square region from $(-5,5)$ to $(5,15)$. All the initial guesses are listed in Table I and plotted as asterisks in Fig. 6. Fig. 7 shows the trace of typical stochastic gradient search starting from No. 1 initial guess. The absolute registration errors for translation in $\mathrm{X}$ and $\mathrm{Y}$ directions are plotted in Fig. 8 (i) and (ii) respectively. Theses error residuals represent the difference between the true and estimated solutions. Although the simplex direct search behaves well for some nonlinear optimization problems [1, 11], unfortunately it succeeds only for experiment No. 3 during our translation registration experiments. Both hill climbing and stochastic gradient search achieves good results, but the mean registration error of stochastic gradient search is less than 0.03 pixel, obviously better than the 0.34 pixel of hill climbing search. During the experiments, the default values of all the control parameters of the search algorithms were adopted based on the initial references. The 500 iterations were fixed in the stochastic gradient search.

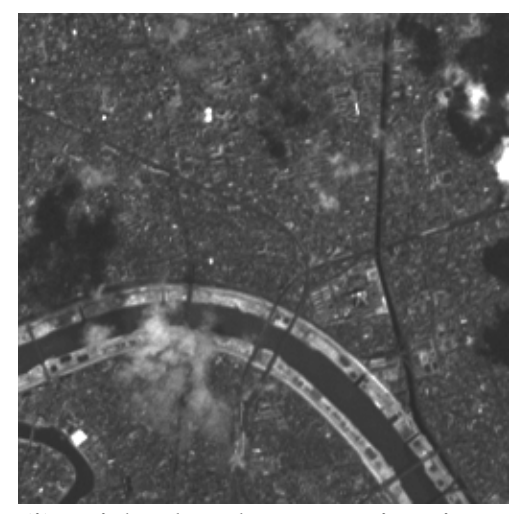

(i) with cloud contamination (Date: 08/23/2001)

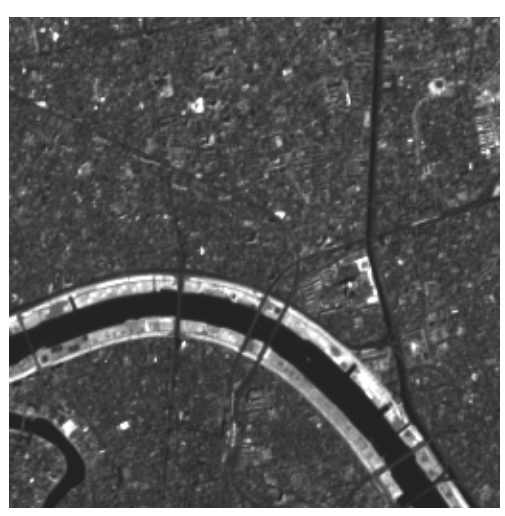

(ii) without cloud contamination (Date: 09/24/2001)

Figure 5: ASTER VNIR image (size: 256x256 pixel$^{2}$; Location: Tokyo bay). 
Table I: Random initial guess for the translation registration.

\begin{tabular}{|c|c|c|}
\hline Experiment No. & X (Pixel) & Y (Pixel) \\
\hline 1 & 10.0030 & 4.2948 \\
\hline 2 & 5.7396 & 2.8396 \\
\hline 3 & 9.8188 & 0.4030 \\
\hline 4 & 10.4194 & 2.1581 \\
\hline 5 & 11.4797 & 4.0608 \\
\hline 6 & 11.6998 & 4.5350 \\
\hline 7 & 13.6334 & -3.7055 \\
\hline 8 & 7.8322 & -4.5914 \\
\hline 9 & 13.5747 & -2.6475 \\
\hline 10 & 7.5768 & -0.9674 \\
\hline
\end{tabular}

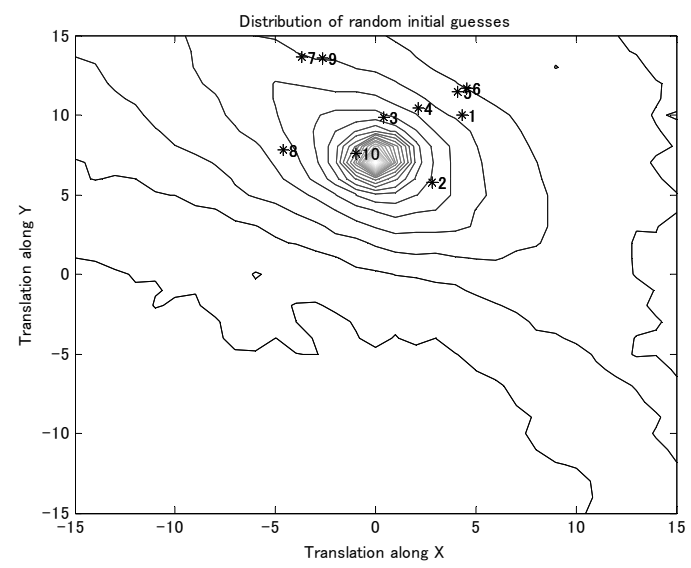

Figure 6: Distribution of initial guesses around the true solution.

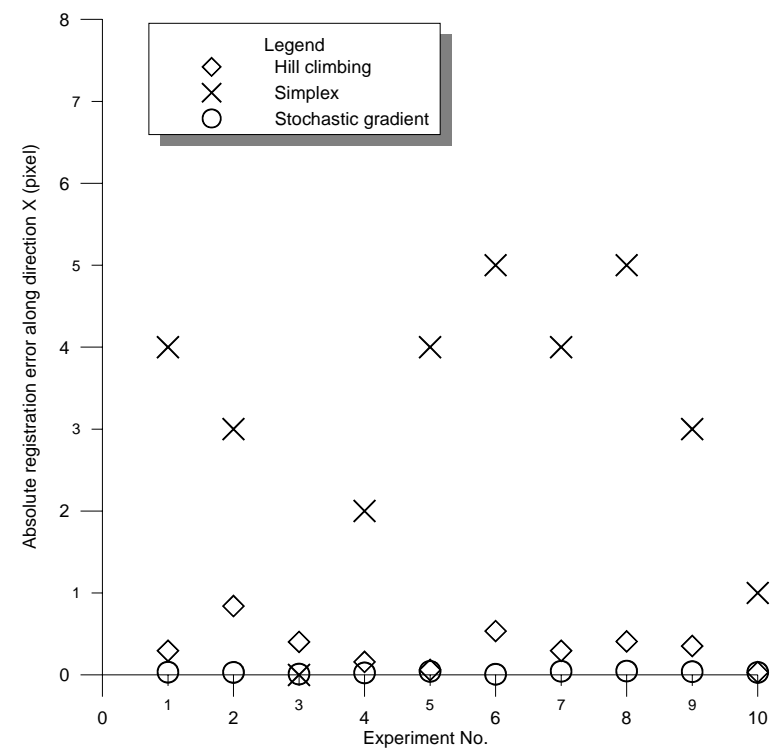

(i) In $\mathrm{X}$ direction

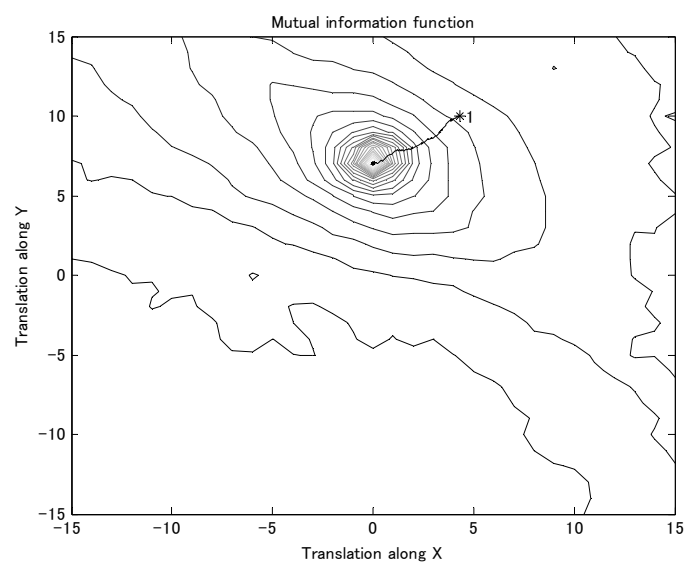

Figure 7: Trace of stochastic gradient search starting from No.1 initial guess.

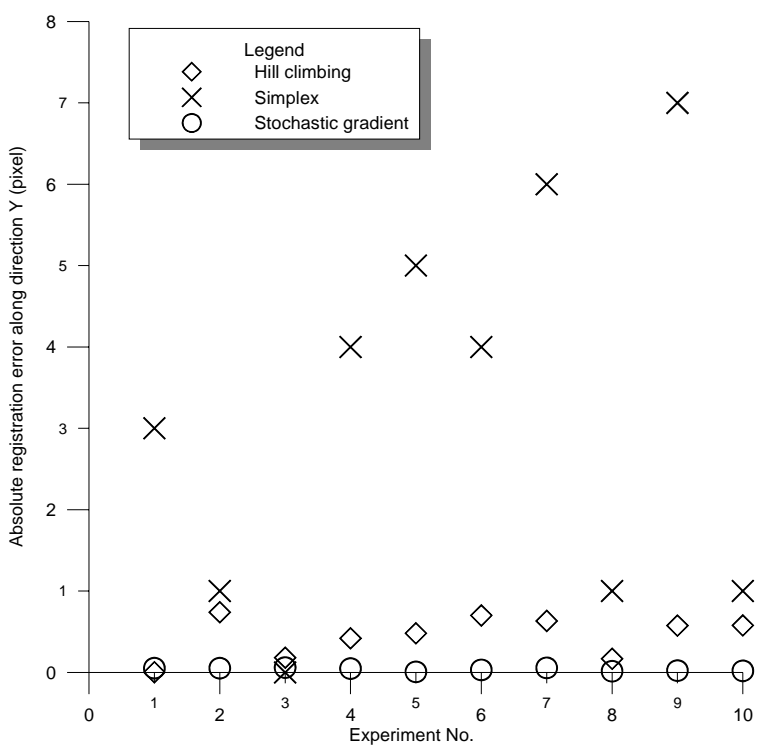

(ii) In Y direction

Figure 8: Absolute registration error for translation in $\mathrm{X}$ and $\mathrm{Y}$ directions. 


\subsection{Affine transformation}

In the third experiment, the stochastic gradient search is used to register the multimodality medical images with the affine transformations. In the experiments, the powerful Brainweb simulated MRI (magnetic resonance imaging) volumes for a normal brain are used. The main advantage to use the simulated MRI database is that the ground truth is known [16].

In the experiments, one triplet of 2D slice of 3D PD (proton density), T2 and T1 MRI volume images along sagittal direction for a normal brain is used. The T2 MRI imaged is transformed in an affine transformation $\hat{T}_{2}^{\text {affine }}$ with $s_{1}=t_{1}=0.1, \theta_{1}=\phi_{1}=5$ degrees, $e_{1}=f_{1}=3$ pixels. The T1 MRI image is transformed in an affine transformation $\hat{T}_{1}^{\text {affine }}$ with $s_{2}=t_{2}=0.2$, $\theta_{2}=\phi_{2}=10$ degrees, $e_{2}=f_{2}=5$ pixels. During the generation process of the transformed T2 and T1 MRI images, the linear interpolation was used. The PD, transformed T2 and transformed T1 MRI images (size: 256x256 pixel$^{2}$ ) are shown in Fig. 9.

The similarity measure $p M I$ in Eq. (8) was used to register the 2D PD and transformed T2 MRI images, and the 2D PD and transformed T1 MRI images in sequence. The initial guess for both experiments is $(0,0,0,0,0,0)$. The trajectories of the stochastic gradient search are plotted in Fig. 10. It is noted that Theta and Phi are plotted in radian. Table II shows the registration results of 2D PD and transformed T2 MRI slices. Table III shows 2D PD and transformed T1 MRI slices. The 10000 iterations were fixed in the search process. The experimental results demonstrate that the stochastic gradient search as a local optimizer is efficient to the multimodality image registration with an affine transformation.

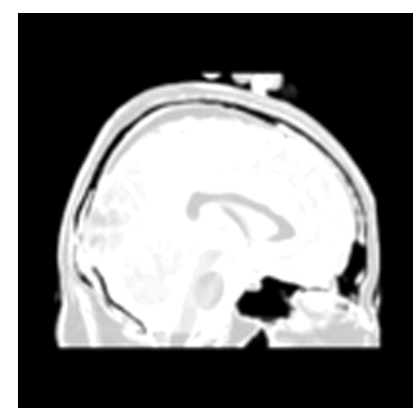

(i) PD

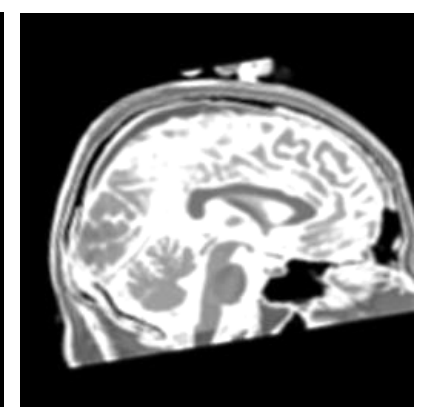

(ii) MRI T2

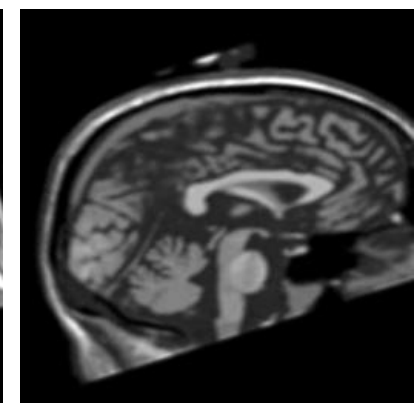

(iii) MRI T1

Figure 9: 2D slice of 3D PD and MRI volumes along sagittal direction for a normal brain.

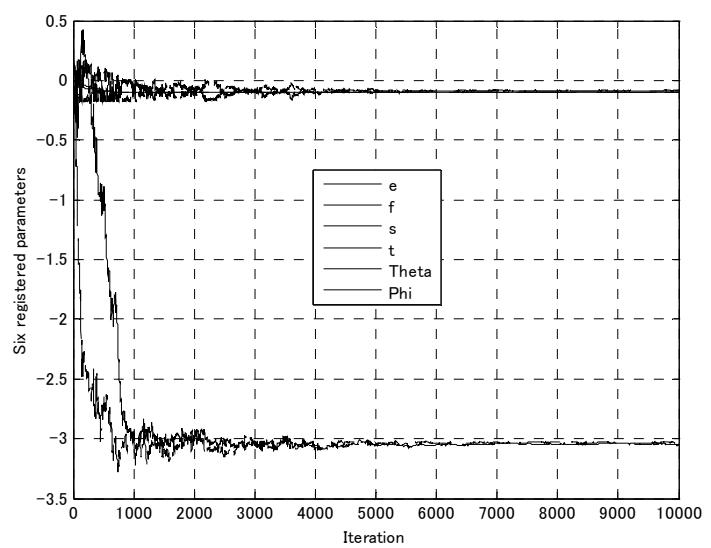

(i) 2D slice PD vs. MRI T2

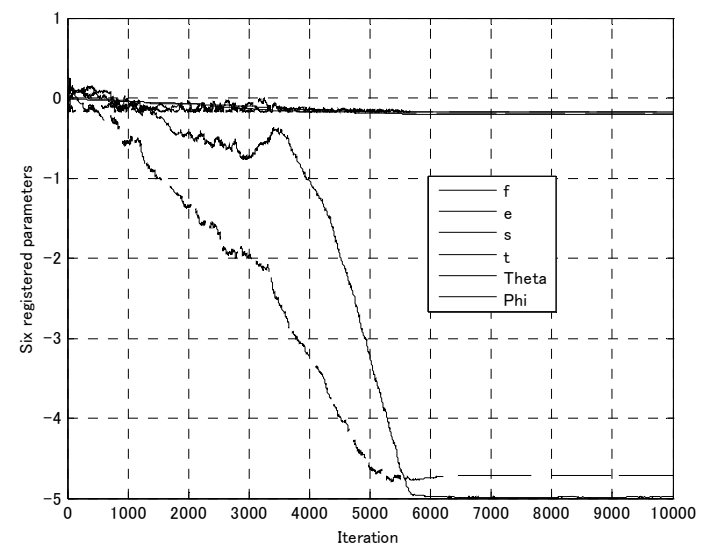

(ii) 2D slice PD vs. MRI T1

Figure 10: Search trajectories for registered affine parameters. 
Table II: Registration results of 2D slice PD and MRI T2.

\begin{tabular}{|c|c|c|}
\hline Affine parameters & Ground truth & Stochastic gradient search \\
\hline$s$ & 0.1 & 0.1 \\
\hline$t$ & 0.1 & 0.1 \\
\hline$\theta$ (Theta) & 5 & 4.9 \\
\hline$\phi(P h i)$ & 5 & 5 \\
\hline$e$ & 3 & 3 \\
\hline$f$ & 3 & 3 \\
\hline
\end{tabular}

Table III: Registration results of 2D slice PD and MRI T1.

\begin{tabular}{|c|c|c|}
\hline Affine parameters & Ground truth & Stochastic gradient search \\
\hline$s$ & 0.2 & 0.2 \\
\hline$t$ & 0.2 & 0.2 \\
\hline$\theta$ (Theta) & 10 & 9.9 \\
\hline$\phi(P h i)$ & 10 & 10 \\
\hline$e$ & 5 & 5 \\
\hline$f$ & 5 & 4.7 \\
\hline
\end{tabular}

\section{CONCLUSIONS \& FUTURE WORK}

An efficient local stochastic gradient search strategy on optimizing the mutual information based similarity measure was firstly and successfully accomplished to simultaneously register the affine transformation for the multimodality images. The experimental results show that in general as a local optimizer the stochastic gradient search yields significant improvements on the optimal solution over the conventional hill climbing and simplex direct search in terms of accuracy and robustness.

As a future work, we plan to develop an adaptive scheme to choose of the control parameters used in the stochastic gradient search for the purpose of multiresolution registration of multiple images.

\section{REFERENCES}

[1] Li, Q.; Sato, I.; Murakami, Y. (2006). Automated image registration using stochastic optimization strategy of mutual information, Proceedings of the international conference on sensing, computing and automation, 2872-2877

[2] Viola, P. A.; Wells III, W. M. (1995). Alignment by maximization of mutual information. Proceedings of the fifth international conference on computer vision, 20-23

[3] Collignon, A.; Maes, F.; Vandermeulen, D.; Suetens, P.; Marchal, G. (1995). Automated multimodality image registration using information theory, Bizais, Y.; Barillot, C.; Di Paola, R. (Editors), Information Processing in Medical Imaging, Kluwer, Dordrecht, 263-274

[4] Press, W. H.; Teukolsky, S. A.; Vetterling, W. T.; Flannery, B. P. (2002). Numerical Recipes in C++: The Art of Scientific Computing (2nd edition), Cambridge University Press, New York

[5] Spall, J. C. (2003). Introduction to Stochastic Search and Optimization: Estimation, Simulation and Control, John Wiley \& Sons, New Jersey

[6] Cole-Rhodes, A. A.; Johnson, K. L.; LeMoigne, J.; Zavorin, I. (2003). Multiresolution registration of remote sensing imagery by optimization of mutual information using a stochastic gradient. IEEE Transactions on Image Processing, Vol. 12, No. 12, 1495-1511

[7] Yoo, T. S. (2004). Insight Into Images, Principles and Practice for Segmentation, Registration, and Image Analysis, A K Peters, Massachusetts 
[8] Mueller, U.; Hesser, J.; Maenner, R. (2005). Optimal parameter choice for automatic fast rigid multimodal registration, Fitzpatrick, J. M.; Reinhardt, J. M. (Editors), Medical Imaging 2005: Image Processing, SPIE, USA, Vol. 5747, 163-169

[9] Cover, T. M.; Thomas, J. A. (1991). Elements of Information Theory, John Wiley \& Sons, New York

[10] Zhang, J.; Rangarajan, A. (2004). Affine image registration using a new information metric, Proceedings of the 2004 IEEE Computer Society Conference on Computer Vision and Pattern Recognition, Vol. 1, 848-855

[11] Chen, H.; Varshney, P. K.; Arora, M. K. (2003). Mutual information based image registration for remote sensing data, International Journal of Remote Sensing, Vol. 24, No. 18, 3701-3706

[12] http://foldoc.org/, accessed on 18-8-2006

[13] Burke, E. K.; Kendall, G. (2005). Search Methodologies: Introductory Tutorials in Optimization and Decision Support Techniques, Springer, New York

[14] Nelder, J. A.; Mead, R. (1965). A simplex method for function minimization, The Computer Journal, Vol. 7, No. 4, 308-313

[15] Li, Q.; Sato, I.; Murakami, Y. (2006). Simultaneous perturbation stochastic approximation algorithm for automated image registration optimization, Proceedings of 2006 IEEE international geoscience and remote sensing symposium \& $27^{\text {th }}$ Canadian symposium on remote sensing, 184187

[16] http://www.bic.mni.mcgill.ca/brainweb/, accessed on 18-8-2006 